\title{
LINEAR WATER WAVES OVER A GENTLY SLOPING BEACH
}

\author{
BY
}

\author{
S. M. SUN AND M. C. SHEN
}

University of Wisconsin, Madison, Wisconsin

\begin{abstract}
The objective of this paper is to justify rigorously the ray method originally developed by Keller [6] for linear water waves over a two-dimensional gently sloping beach. The approximate formula for eigenvalues of the linear water wave problem and the uniform ray method expansion at and near a shoreline are all consequences of the justification.
\end{abstract}

1. Introduction. The problem of linear water waves over a uniformly sloping beach was completely solved by Ursell [1], Peters [2], and Roseau [3]. An interesting account of the history and a detailed derivation of the solutions of the problem can be found in Stoker [4]. Let $\omega^{2}$ be the eigenvalue parameter in the time-reduced problem and let $k$ be the nondimensional wave number along the shoreline. For $0<k \leq 1$, there is a continuous spectrum of $\omega^{2}$ and the solution behaves like a progressive wave at infinity. For $k>1$, there is a discrete spectrum of $\omega^{2}$, which consists of finitely many eigenvalues, and the number of eigenvalues increases as the slope of the beach decreases. The corresponding eigenfunctions decay exponentially in the direction perpendicular to the shoreline. For $0<k \leq 1$ the solution with a finite amplitude at the shoreline behaves like the zeroth-order Bessel function of the first kind. The same behavior of a solution to the shallow water equations was also discovered [5]. For the problem of linear water waves over a general variable bottom, Keller [6] developed a ray method to solve the time-reduced problem under the conditions that $\omega$ is large and the bottom topography changes slowly. In this method a wave-like solution consisting of an amplitude function and a phase function is assumed. The phase function satisfies an eiconal equation which can be solved by the method of characteristics and yields a family of rays. Along each ray a transport equation can be integrated to determine the square of the amplitude function to the first-order approximation. This work was extended to the calculation of the eigenvalues of the water wave problem by Shen, Meyer, and Keller [7] on the basis of Keller and Rubinow [8]. The ray method expansion fails at a shoreline and a caustic where the amplitude function becomes infinite. Following some ideas due to Kravtsov [9] and Ludwig [10], a uniform ray method using a solution of a comparison equation

Received October 31, 1991.

1991 Mathematics Subject Classification. Primary 76B15; Secondary 35P20.

(C)1994 Brown University 
was constructed by Shen and Keller [11]. The comparison equation for the uniform expansion at a shoreline is just the first-order Bessel equation as expected. Up to now the ray methods developed are formal in the sense that the expansions in terms of the negative integral powers of a large parameter are only assumed but not justified, even though the approximate solutions obtained by the ray method compare favorably with the existing exact solutions for a uniformly sloping beach.

The objective of this paper is to provide a rigorous justification of the ray method for linear water waves over a two-dimensional gently sloping beach with a nonzero slope at the shoreline. We show that the wave function determined by the ray method is a uniform approximate solution of the exact linear equations governing the motion of the fluid for the beach with small slope. Also each eigenvalue derived from the ray method is close to the spectrum of the linear operator of the water wave problem. The point of departure is an integral representation due to Zhevandrov [12] motivated by the original results of Miles [13]. Some ideas due to Maslov and Fedoriuk [14] for a caustic in the WKB method will be used for the justification. However, the singularity at a shoreline is quite different from that at a caustic. In fact, a function equivalent to the optical refraction index in linear water wave problems becomes infinite at a shoreline. A suitable contour must be chosen so that the integral representation is meaningful.

We formulate the problem in Sec. 2. An integral representation of the solution of the problem is constructed in Sec. 3. An approximate expression to the integral representation is verified in Sec. 4., where an approximate spectral formula for the eigenvalues in [7] is also obtained. In Sec. 5, we justify the ray method expansion away from a shoreline [6] and the uniform ray method expansion near and at a shoreline [11]. A discussion about the validity of the spectral formula is also given.

2. Formulation. We consider a two-dimensional gently sloping beach with a nonzero slope at the shoreline. A coordinate system $X, Y, Z$ is chosen so that the plane $Z=0$ coincides with the equilibrium surface and $Z$ increases upward. The depth of the seabed is $Z=-H(X)$, a function of $X$ only. The shoreline corresponds to $Z=0, X=0$, and $H(0)=0$. The linear theory of water waves is based on the equations in [4]:

$$
\begin{aligned}
\Phi_{X X}+\Phi_{Y Y}+\Phi_{Z Z}=0 & \text { in } 0>Z>-H(X), \\
\Phi_{t t}+g \Phi_{Z}=0 & \text { at } Z=0, \\
\Phi_{Z}+H_{X} \Phi_{X}=0 & \text { at } Z=-H(X),
\end{aligned}
$$

where $\Phi(X, Y, Z, t)$ is the velocity potential, $t$ is time, and $g$ is the constant gravitational acceleration.

Assume that the slope is small and negative, that is, $H^{\prime}(X) \ll 1$ and $H^{\prime}(X)>0$, which generally characterizes oceanographical topographies, and the function $H(X)$ has the form $H(X)=k^{-1} h(x)$ where $x=\varepsilon k X, \varepsilon=H^{\prime}(0)<<1$, and $k$ is the wave number along the shore; let

$$
\Phi(X, Y, Z, t)=\exp (i(\omega t-k y)) \phi(x, z, \varepsilon),
$$


with $y=Y$ and $z=k Z$. Then Eqs. (1)-(3) become

$$
\begin{aligned}
\phi_{z z}+\varepsilon^{2} \phi_{x x}-\phi=0 & \text { in } 0>z>-h(x), \\
\phi_{z}=\lambda \phi & \text { at } z=0, \\
\phi_{z}+\varepsilon^{2} h_{x} \phi_{x}=0 & \text { at } z=-h(x),
\end{aligned}
$$

where $\lambda=\omega^{2} /(g k)$.

The approximate integral representation of a solution satisfying Eqs. (4) and (5) due to Zhevandrov [12] assumes the form

$$
\phi_{1}(x, z)=\int_{C} \exp (i p x / \varepsilon)\left(\cosh (\kappa z)+\lambda \kappa^{-1} \sinh (\kappa z)\right) f(p) d p,
$$

where $\kappa=\left(p^{2}+1\right)^{1 / 2}, f(p)$ is an unknown function, and $C$ is a contour in the complex $p$-plane. Equation (7) can be obtained formally as follows. Extend Eqs. (4) and (5) evenly to $x \leq 0$ and take a $(1 / \varepsilon)$-Fourier transformation [14] of Eqs. (4) and (5) with respect to $x$ to obtain

$$
\begin{aligned}
\hat{\phi}_{z z}-\left(p^{2}+1\right) \hat{\phi}=0 & \text { in } 0>z>-\hat{h}(p), \\
\hat{\phi}_{z}-\lambda \hat{\phi}=0 & \text { at } z=0,
\end{aligned}
$$

where

$$
\hat{\phi}(p, z)=\left(\frac{1}{2 \pi \varepsilon i}\right) \int \phi(x, z) \exp (-i p x / \varepsilon) d x
$$

Therefore,

$$
\hat{\phi}(p, z)=f(p)\left(\cosh (\kappa z)+\lambda \kappa^{-1} \sinh (\kappa z)\right)
$$

and

$$
\phi(x, z)=\left(\frac{-1}{2 \pi \varepsilon i}\right) \int \hat{\phi}(p, z) \exp (i p x / \varepsilon) d p,
$$

which equals Eq. (7) formally up to a multiplicative constant.

From Eq. (7), $f(p)$ cannot be chosen to have compact support in $p$ if the intent is to study the behavior of the solution near a shoreline. The reason will be given later. Thus, by the exponential growth of the functions $\cosh (\kappa z)$ and $\sinh (\kappa z)$ a proper contour $C$ must be chosen so that Eq. (7) is meaningful. From now on we assume $f(p)$ is bounded and $C$ is the contour shown in Fig. 1 (see p. 246), where $R$ is a large positive number to be chosen later.

Therefore, $\phi_{1}(x, z, \varepsilon)$ in Eq. (7) is well defined for $x>0$ since $f(p)$ is bounded. Obviously by substituting Eq. (7) for $\phi_{1}(x, z)$ in the equations, it is easy to see that Eqs. (4) and (5) are satisfied. By Eq. (6), we need

$$
\begin{aligned}
\int_{C} \exp (i p x / \varepsilon)(\lambda & \cosh (\kappa h(x))-\kappa \sinh (\kappa h(x)) \\
& \left.+i \varepsilon h_{x}(x) p\left(\cosh (\kappa h(x))-\lambda \kappa^{-1} \sinh (\kappa h(x))\right)\right) f(p) d p=0 .
\end{aligned}
$$

But it is impossible to find $f(p)$ so that Eq. (8) is satisfied. Instead we construct $f(p)$ so that the left-hand side of Eq. (8) is small up to some order of $\varepsilon$. Then $\phi_{1}(x, z)$ defined in Eq. (7) is an approximate solution of Eqs. (4)-(6) for small $\varepsilon$. 


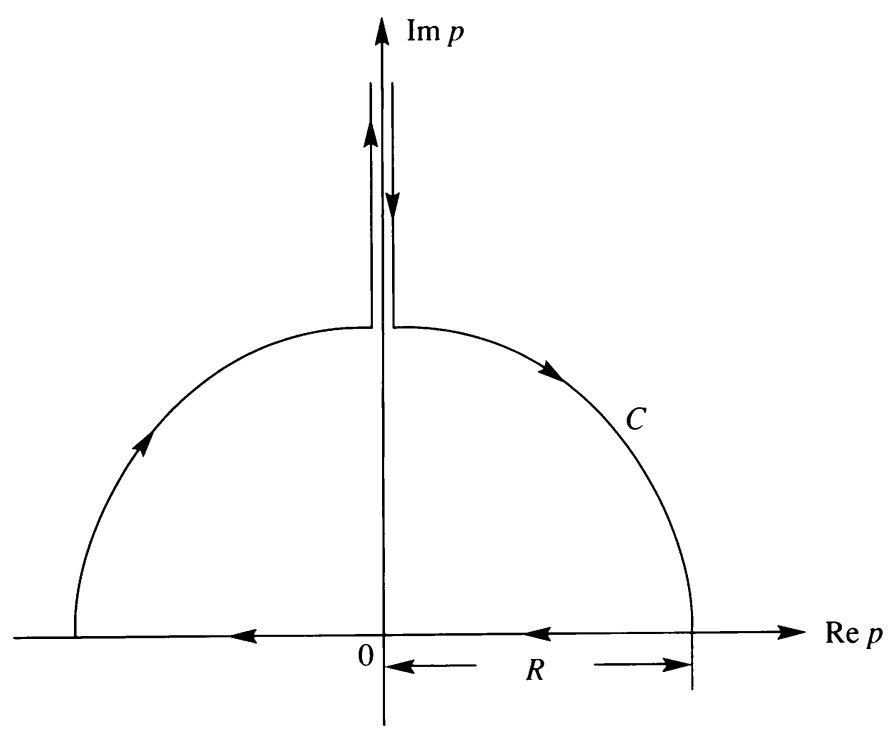

Fig. 1. The contour $C$

3. Construction of the solution. Let us denote the left-hand side of Eq. (8) as $I(x)$ and

$$
\begin{aligned}
L(x, p) & =\lambda \cosh (\kappa h(x))-\kappa \sinh (\kappa h(x)), \\
M(x, p) & =h_{x}(x) p\left(\cosh (\kappa h(x))-\lambda \kappa^{-1} \sinh (\kappa h(x))\right) .
\end{aligned}
$$

Thus,

$$
I(x)=\int_{C} \exp (i p x / \varepsilon)(L(x, p)+i \varepsilon M(x, p)) f(p) d p .
$$

Following the ideas from Maslov and Fedoriuk [14] we let

$$
f(p)=a(p) \exp (-i S(p) / \varepsilon),
$$

and Eq. (9) becomes

$$
I(x)=\int_{C} \exp (i(p x-S(p)) / \varepsilon)(L(x, p)+i \varepsilon M(x, p)) a(p) d p,
$$

where $a(p)$ and $S(p)$ are bounded. By integration by parts and noting that $x>0$ and $\exp (i p x) \rightarrow 0$ exponentially as $p \rightarrow \infty$ along $C$, we have

$$
\begin{aligned}
\int_{C} \exp (i(p x-S(p)) / \varepsilon) F(x, p) d p \\
=\int_{C} \exp (i(p x-S(p)) / \varepsilon) F\left(S_{p}, p\right) d p \\
\quad+i \varepsilon \int_{C} \exp (i(p x-S(p)) / \varepsilon) \mathscr{T}(F)(x, p) d p,
\end{aligned}
$$


where $F(x, p)$ is sufficiently smooth in $x$ and analytic in $p$ on $C$, and

$$
\begin{aligned}
\mathscr{T}(F)(x, p) & =\frac{\partial}{\partial p}\left(\frac{F(x, p)-F\left(S_{p}, p\right)}{x-S_{p}}\right) \\
& =\int_{0}^{1}\left(\left(1-\theta_{1}\right) S_{p p} F_{x x}+F_{x p}\right)\left(\theta_{1} x+\left(1-\theta_{1}\right) S_{p}, p\right) d \theta_{1} .
\end{aligned}
$$

By using Eq. (10) several times, we have

$$
\begin{aligned}
I(x)= & \int_{C} \exp (i(p x-S(p)) / \varepsilon) \\
& \times\left(a(p) L\left(S_{p}, p\right)+i \varepsilon \mathscr{T}(a L)\left(S_{p}, p\right)-\varepsilon^{2} \mathscr{T}^{2}(a L)(x, p)\right. \\
& \left.+i \varepsilon M\left(S_{p}, p\right) a(p)-\varepsilon^{2} \mathscr{T}(a M)(x, p)\right) d p,
\end{aligned}
$$

where

$$
\begin{aligned}
\mathscr{T}^{2}(f)(x, p)= & \int_{0}^{1} \int_{0}^{1}\left(\theta_{1}^{2}\left(1-\theta_{1}\right)\left(1-\theta_{2}\right) S_{p p}^{2} f_{x x x x}+\theta_{1}^{2}\left(1-\theta_{2}\right) S_{p p} f_{x x x p}\right. \\
& +\theta_{1}\left(1-\theta_{1}\right)^{2} S_{p p}^{2} f_{x x x x}+\theta_{1}\left(1-\theta_{1}\right) S_{p p p} f_{x x x}+2\left(1-\theta_{1}\right) \theta_{1} S_{p p} f_{x x x p} \\
& \left.+\theta_{1} f_{x x p p}\right)\left(\theta_{1} \theta_{2}\left(x-S_{p}\right)+S_{p}, p\right) d \theta_{1} d \theta_{2} .
\end{aligned}
$$

In Eq. (11), the terms of zeroth- and first-orders of $\varepsilon$ are zero if we let

$$
\begin{gathered}
L\left(S_{p}, p\right)=0, \\
\mathscr{T}(a L)\left(S_{p}, p\right)+M\left(S_{p}, p\right) a(p)=0 .
\end{gathered}
$$

First let us solve Eq. (12) for $S(p)$ by the method of characteristics. Let $P=S_{p}$. Then

$$
\begin{aligned}
& \frac{d p}{d \sigma}=h_{x}(P)\left(\lambda^{2}-\left(p^{2}+1\right)\right) \cosh \left(\left(p^{2}+1\right)^{1 / 2} h(P)\right), \\
& \frac{d P}{d \sigma}=p \cosh \left(\left(p^{2}+1\right)^{1 / 2} h(P)\right)\left(h(P)+\lambda(1-\lambda h(P))\left(p^{2}+1\right)^{-1}\right), \\
& \frac{d S}{d \sigma}=P h_{x}(P)\left(\lambda^{2}-\left(p^{2}+1\right)\right) \cosh \left(\left(p^{2}+1\right)^{1 / 2} h(P)\right),
\end{aligned}
$$

with initial condition $\left(p_{0}, P_{0}, S_{0}\right)$ at $\sigma=\sigma_{0}$ satisfying $L\left(P_{0}, p_{0}\right)=0$. But to solve $P$ from $L(P, p)=0$ for every real $p$, it is straightforward to show that $\lambda$ must be greater than zero and less than $\tanh h(\infty)<1$. Then if $p$ is real or $|p|$ is large, $\lambda^{2}-\left(p^{2}+1\right) \neq 0$ and from Eq. (14) we can use $p$ as a parameter instead of $\sigma$. Thus we let $p_{0}=0$ and $S=S_{0}$ at $p_{0}=0$.

Then Eqs. (14)-(16) become

$$
\begin{gathered}
\frac{d P}{d p}=p\left(h(P)-\lambda^{2} h(P)\left(p^{2}+1\right)^{-1}+\lambda\left(p^{2}+1\right)^{-1}\right)\left(h_{x}(P)\left(\lambda^{2}-\left(p^{2}+1\right)\right)\right)^{-1}, \\
\frac{d S}{d p}=P .
\end{gathered}
$$


It is more convenient to solve $L(P, p)=0,(17)$ and (18) and we have

$$
\begin{aligned}
h(P) & =\frac{1}{2}\left(p^{2}+1\right)^{-1 / 2} \ln \left(\left(\left(p^{2}+1\right)^{1 / 2}+\lambda\right) /\left(\left(p^{2}+1\right)^{1 / 2}-\lambda\right)\right) \\
& \equiv I I(p) .
\end{aligned}
$$

The logarithm in Eqs. (19) has no singularity since $\lambda<\tanh h(\infty)<1$. For large $p$, it is not difficult to show that $I I(p)$ is an analytic function of $p$. If we assume that $h(P)$ is analytic near $P=0$ and $h^{\prime}(0) \neq 0$, then for large $|p|$,

$$
P=h^{-1}(I I(p))
$$

is an analytic function for large $p$ where $h^{-1}$ is the inverse function of $h$ which is well defined since $h^{\prime}(x)>0$ for $x>0$ by the assumption $H^{\prime}(x)>0$ for $H(x)$. Therefore, by $P(p) \sim O\left(p^{-2}\right)$ as $|p| \rightarrow \infty$,

$$
S(p)=\int_{0}^{p} P(p) d p+S_{0},
$$

with $S(p)$ analytic for large $|p|$. Thus we have solved Eq. (12) for $S(p)$ with complex $p$.

Next let us solve Eq. (13). We can rewrite Eq. (13) as

$$
\frac{1}{2} a(p) L_{x x}\left(S_{p}, p\right) S_{p p}+a_{p}(p) L_{x}\left(S_{p}, p\right)+a(p) L_{x p}\left(S_{p}, p\right)+M\left(S_{p}, p\right)=0 \text {. }
$$

By some straightforward calculation, Eq. (22) can be expressed as

$$
\begin{aligned}
a_{p}(p) & \left(\lambda^{2}-\left(p^{2}+1\right)\right) h_{x}\left(S_{p}\right) \\
= & a(p)\left(-\frac{1}{2} S_{p p} h_{x x}\left(S_{p}\right)\left(\lambda^{2}-\left(p^{2}+1\right)\right)-p h_{x}\left(S_{p}\right)\left(\lambda^{2}\left(p^{2}+1\right)^{-1}-2\right)\right. \\
& \left.-p h_{x}\left(S_{p}\right)\left(1-\lambda^{2}\left(p^{2}+1\right)^{-1}\right)\right) \\
= & a(p)\left(-\frac{1}{2} S_{p p} h_{x x}\left(S_{p}\right)\left(\lambda^{2}-\left(p^{2}+1\right)\right)+p h_{x}\left(S_{p}\right)\right) .
\end{aligned}
$$

Then

$$
\begin{aligned}
a(p) & =a_{0}\left(p^{2}+1-\lambda^{2}\right)^{-1 / 2}\left(h_{x}\left(S_{p}\right)\right)^{-1 / 2} \\
& =a_{0}\left(p^{2}+1\right)^{-1 / 2}\left(1-\lambda^{2}\left(p^{2}+1\right)^{-1}\right)^{-1 / 2}\left(h_{x}\left(S_{p}\right)\right)^{-1 / 2},
\end{aligned}
$$

since $\left(1-\lambda^{2}\left(p^{2}+1\right)^{-1}\right)^{-1 / 2}\left(h_{x}\left(S_{p}\right)\right)^{-1 / 2}$ is an analytic function for large $p$. Note that $a(p)$ must be of the form (23) if Eq. (13) holds and from $L(x, p)=0, p$ must be large when $x$ is small. Thus if we need a solution near $x=0$, we cannot assume that $a(p)$ has compact support in $p$.

From Eqs. (21) and (23), we have

$$
\begin{aligned}
\phi_{1}(x, z)= & \int_{C} \exp (i p x / \varepsilon)\left(\cosh (\kappa z)+\lambda \kappa^{-1} \sinh (\kappa z)\right) \\
& \times\left(\exp \left(-i\left(\int_{0}^{p} P(p) d p+S_{0}\right) / \varepsilon\right)\right) a_{0}\left(p^{2}+1\right)^{-1 / 2} A(p) d p \\
= & \int_{C} I I I(p) \exp \left(-i\left(\int_{0}^{p} P(p) d p\right) / \varepsilon\right)\left(p^{2}+1\right)^{-1 / 2} d p,
\end{aligned}
$$

where $A(p)=\left(1-\lambda^{2}\left(p^{2}+1\right)^{-1}\right)^{-1 / 2}\left(h_{x}\left(S_{p}\right)\right)^{-1 / 2}$. Since we know that $h(x)$ is analytic near $x=0$, we see that for $p$ complex with large $p$, the factors inside the integral 
are all analytic except $\left(p^{2}+1\right)^{-1 / 2}$ which is analytic for $\operatorname{Im} p \geq 0$ with a slit from $p=i$ to $p=i \infty$ along the imaginary axis. Therefore, $\phi_{1}(x, z)$ is well defined for $x>0$ and $R$ can be any real positive number greater than $R^{\prime}$, where for $|p| \geq R^{\prime}$ and $\operatorname{Im} p \geq 0$, the integrand in Eq. (24) is analytic.

4. Approximate solution. Now we need to verify that $\phi_{1}(x, z)$ defined by Eq. (24) is an approximate solution of Eqs. (4)-(6). Before doing that, we first show that we can choose some $\lambda$ so that $\phi_{1}(x, z)$ is finite as $x \rightarrow 0^{+}$.

As we have already seen, the convergence of the integral in Eq. (24) depends on $x>0$. If we let

$$
\begin{aligned}
& C_{I}^{+}=\left\{p=p_{r}+i p_{i} \mid p_{r}=0^{+} \text {and } p_{i} \geq R\right\}, \\
& C_{I}^{-}=\left\{p=p_{r}+i p_{i} \mid p_{r}=0^{-} \text {and } p_{i} \geq R\right\},
\end{aligned}
$$

$C_{I}=C_{I}^{+} \cup C_{I}^{-}$, and $C_{R}=C \backslash C_{I}$, then from Eq. (24)

$\phi_{1}(x, z)=\left(\int_{C_{I}^{+}}+\int_{C_{I}^{-}}+\int_{C_{R}}\right)\left(I I I(p) \exp \left(-i\left(\int_{0}^{p} P(p) d p\right) / \varepsilon\right)\left(p^{2}+1\right)^{-1 / 2}\right) d p$.

The first two terms of Eq. (25) are

$$
\begin{aligned}
I V(x, z)= & \left(\int_{0^{-}+R i}^{0^{-}+\infty i}+\int_{0^{+}+\infty i}^{0^{+}+R i}\right)\left(I I I(p) \exp \left(-i\left(\int_{0}^{p} P(p) d p\right) / \varepsilon\right)\left(p^{2}+1\right)^{-1 / 2}\right) d p \\
= & \int_{0^{-}+R i}^{0^{-}+\infty i} I I I(p) \exp \left(-i\left(\left(\int_{-\infty}^{p}+\int_{0}^{-\infty}\right) P(p) d p\right) / \varepsilon\right)\left(p^{2}+1\right)^{-1 / 2} d p \\
& +\int_{0^{+}+\infty i}^{0^{+}+R i} I I I(p) \exp \left(-i\left(\left(\int_{+\infty}^{p}+\int_{0}^{+\infty}\right) P(p) d p\right) / \varepsilon\right)\left(p^{2}+1\right)^{-1 / 2} d p .
\end{aligned}
$$

Since $P(p)$ is even in $p$ and analytic in $p$ for $p$ large,

$$
\begin{aligned}
I V(x, z)= & \int_{0^{+}+\infty i}^{0^{+}+R i} I I I(p) \exp \left(-i\left(\int_{+\infty}^{p} P(p) d p\right) / \varepsilon\right)\left(p^{2}+1\right)^{-1 / 2} \\
& \times\left(\exp \left(-i\left(\int_{0}^{+\infty} P(p) d p\right) / \varepsilon\right)\right. \\
& \left.-\exp \left(-i\left(\left(\int_{0}^{-\infty} P(p) d p\right) / \varepsilon+\alpha\right)\right)\right) d p,
\end{aligned}
$$

where $\alpha=(2 n+1) \pi$ for an integer $n$ since $\left(p^{2}+1\right)^{-1 / 2}$ has a branch point at $p=i$. Therefore, if we let

$$
\int_{-\infty}^{+\infty} P(p) d p=\alpha \varepsilon
$$

which determines the values of $\lambda$, then $I V(x, z) \equiv 0$ for all large $R$ and Eq. (25) becomes

$$
\begin{aligned}
\phi_{1}(x, z)= & \int_{C_{R}} \exp \left(i\left(p x-\left(\int_{0}^{p} P(p) d p+S_{0}\right)\right) / \varepsilon\right) a_{0}\left(p^{2}+1\right)^{-1 / 2} A(p) \\
& \times\left(\cosh (\kappa z)+\lambda \kappa^{-1} \sinh (\kappa z)\right) d p .
\end{aligned}
$$


From Eq. (27), we see that as $x \rightarrow 0, \phi_{1}(x, z)$ is finite, and $\phi_{1}(x, z)$ is independent of $R$ for large $p$. In the following we always assume that Eq. (26) holds.

Now we show that $\phi_{1}(x, z)$ defined in Eq. (24) is an approximate solution of Eqs. (4)-(6). Equations (4) and (5) are satisfied obviously. We substitute Eq. (24) for $\phi_{1}$ in Eq. (6) to obtain Eq. (11). By our construction of $S(p)$ and $a(p)$, we have

$$
\begin{aligned}
I(x)= & -\varepsilon^{2} \int_{C}\left(\mathscr{T}^{2}(a L)(x, p)+\mathscr{T}(a M)(x, p)\right) \\
& \times \exp \left(i\left(p x-\left(\int_{0}^{p} P(p) d p+S_{0}\right)\right) / \varepsilon\right) d p .
\end{aligned}
$$

Let $\mathscr{T}^{2}(a L)+\mathscr{T}(a M)=B(x, p)\left(p^{2}+1\right)^{-1 / 2}$. It is not difficult to check term-byterm in $B(x, p)$ that $B(x, p)$ is analytic in $p$ for large $|p|$ and if $x=C_{1}|p|^{-2}$, then $|B(x, p)| \leq C_{2}$ where $C_{1}$ is any positive constant, $|p|$ is large, and $C_{2}$ only depends on $C_{1}$. Thus by Eq. (26), the integrals in Eq. (28) from $0^{-}+i R$ to $0^{-}+i \infty$ and from $0^{+}+i \infty$ to $0^{+}+i R$ cancel each other and Eq. (28) becomes

$$
I(x)=-\varepsilon^{2} \int_{\pi}^{0} D\left(x, \operatorname{Re}^{i \theta}\right) R i e^{i \theta} d \theta-\varepsilon^{2} \int_{-R}^{R} D(x, p) d p,
$$

where $D(x, p)=B(x, p)\left(p^{2}+1\right)^{-1 / 2} \exp \left(i\left(p x-\left(\int_{0}^{p} P(p) d p+S_{0}\right)\right) / \varepsilon\right)$. But $P(p) \sim$ $d_{1} / p^{2}$ for $|p|$ large where $d_{1}$ is a positive constant; then

$$
\int_{0}^{p} p(p) d p \sim d_{2}-\frac{d_{1}}{|p|}, \quad d_{2} \text { is real }
$$

and if $p=p_{r}+i p_{i}$ with $p_{r}, p_{i}$ real,

$$
\begin{aligned}
p x-\left(\int_{0}^{p} P(p) d p+S_{0}\right)= & p x+\left(d_{1} / p\right)(1+O(1 / p))-\left(d_{2}+S_{0}\right) \\
= & p_{r}\left(x+d_{1}|p|^{-2}(1+O(1 /|p|))\right) \\
& +i p_{i}\left(x-d_{1}|p|^{-2}(1+O(1 /|p|))\right)-\left(d_{2}+S_{0}\right) .
\end{aligned}
$$

Thus if we let $x R^{2}=d_{3}$ with $1-\left(2 d_{1} / d_{3}\right)>1 / 2$ in Eq. (29), then for large $|p|$,

$$
\begin{aligned}
\left|-\varepsilon^{2} \int_{\pi}^{0} D\left(x, R e^{i \theta}\right) R i e^{i \theta} d \theta\right| & \leq C_{2} \varepsilon^{2} \int_{0}^{\pi} \exp (-(R x / 2) \sin \theta / \varepsilon) d \theta \\
& \leq\left(2 c_{2} \pi \varepsilon^{3} / R x\right)(1-\exp (-R x / 2 \varepsilon)) .
\end{aligned}
$$

Also if $p$ is real, then $B(x, p)$ is even for $p$ and

$$
\begin{aligned}
\left|\int_{-R}^{R} D(x, p) d p\right| \leq & \mid \int_{0}^{R} B(x, p)\left(p^{2}+1\right)^{-1 / 2} \\
& \times\left(\exp \left(i\left(p x-\left(\int_{0}^{p} P(p) d p+S_{0}\right)\right) / \varepsilon\right)\right. \\
& \left.+\exp \left(i\left(-p x+\int_{0}^{p} P(p) d p-S_{0}\right) / \varepsilon\right)\right) d p \mid,
\end{aligned}
$$


for $x p^{2} \leq d_{3}$ and $B(x, p)=C_{5}+B_{1}(x, p)\left(p^{2}+1\right)^{-1 / 2}$ where $B_{1}(x, p)$ is bounded and $C_{5}$ is a fixed constant. Thus,

$$
\begin{aligned}
\left|\int_{-R}^{R} D(x, p) d p\right| \leq & \left|\int_{0}^{R} C_{5}\left(p^{2}+1\right)^{-1 / 2} \cos \left(\left(p x-\int_{0}^{p} P(p) d p\right) / \varepsilon\right) d p\right| \\
& +\left|\int_{0}^{R} B_{1}(x, p)\left(p^{2}+1\right)^{-1} \cos \left(\left(p x-\int_{0}^{p} P(p) d p\right) / \varepsilon\right) d p\right| .
\end{aligned}
$$

Since $B_{1}(x, p)$ is bounded in $[0, R]$ and the critical point of $p x-\int_{0}^{p} P(p) d p$ is in $[0, R]$, by the stationary phase method we have

$$
\left|\int_{0}^{R} B_{1}(x, p)\left(p^{2}+1\right)^{-1} \cos \left(\left(p x-\int_{0}^{p} P(p) d p\right) / \varepsilon\right) d p\right| \leq C_{6} \varepsilon^{1 / 2}
$$

where $C_{6}$ is a fixed constant. Now we consider

$$
\begin{aligned}
|J| & =\left|\int_{0}^{R}\left(p^{2}+1\right)^{-1 / 2} \cos \left(\left(p x-\int_{0}^{p} P(p) d p\right) / \varepsilon\right) d p\right| \\
& =\mid \int_{0}^{d_{3}^{1 / 2}}\left(q^{2}+x\right)^{-1 / 2} \cos \left(\left(x^{1 / 2} / \varepsilon\right)\left(q+x^{-1 / 2} \int_{0}^{q x^{-1 / 2}} P(p) d p\right)\right) d q \\
& =\left|\int_{0}^{d_{3}^{1 / 2}} J_{1}(x, q) d q\right| \\
& \leq\left|\int_{0}^{\alpha_{1} x^{1 / 2}} J_{1}(x, q) d q\right|+\left|\int_{\alpha_{1} x^{1 / 2}}^{\beta} J_{1}(x, q) d q\right|+\left|\int_{\beta}^{d_{3}^{1 / 2}} J_{1}(x, q) d q\right|,
\end{aligned}
$$

where $\alpha_{1}$ is large and $\beta$ is small, and they are fixed numbers to be chosen. Choose $\alpha_{1}$ so large that when $p \geq \alpha_{1}$

$$
P(p)=\left(d_{1} / p^{2}\right)(1+O(1 / p))
$$

with $1+O\left(p^{-1}\right) \geq \frac{1}{2}$ and

$$
\int_{p}^{+\infty} P(p) d p=\left(d_{1} / p\right)\left(1+O\left(p^{-1}\right)\right) .
$$

Also let

$$
\tau^{-1}=q-x^{-1 / 2} \int_{q x^{-1 / 2}}^{+\infty} P(p) d p=q+\left(d_{1} / q\right)\left(1+O\left(x^{1 / 2} q^{-1}\right)\right)
$$

if $x^{-1 / 2} q \geq \alpha_{1}$ and $\beta$ is small so that $d \tau / d q \geq K>0$ for $\alpha_{1} x^{1 / 2} \leq q \leq \beta$ where $K$ is a fixed constant. After having found $\alpha_{1}$ and $\beta$, we have $q=Q(\tau)$ and

$$
\begin{aligned}
\left|\int_{\alpha_{1} x^{1 / 2}}^{\beta} J_{1}(x, q) d q\right|= & \mid \int_{\tau_{0}}^{\tau_{1}} Q^{\prime}(\tau)\left(Q^{2}(\tau)+x\right)^{-1 / 2} \\
& \times \cos \left(\left(x^{1 / 2} / \varepsilon\right)\left(\tau^{-1}+x^{-1 / 2} \int_{0}^{+\infty} P(p) d p\right)\right) d \tau \mid,
\end{aligned}
$$


where

$$
\begin{aligned}
& \tau_{0}=x^{1 / 2}\left(\alpha_{1} x-\int_{\alpha_{1}}^{+\infty} P(p) d p\right)^{-1}, \\
& \tau_{1}=\left(\beta-x^{-1 / 2} \int_{\beta x^{-1 / 2}}^{+\infty} P(p) d p\right)^{-1},
\end{aligned}
$$

$Q^{\prime}(\tau)$ is uniformly bounded for $\tau_{0} \geq \tau \geq \tau_{1}$ and small $x$, and $\tau_{0}=O\left(x^{1 / 2}\right)$. Also for small $\tau$,

$$
Q(\tau)=\left(\tau / d_{1}\right)(1+O(\tau)), \quad Q^{\prime}(\tau)=\left(1 / d_{1}\right)(1+O(\tau))
$$

By Eq. (26),

$$
\begin{aligned}
\left|\int_{\alpha_{1} x^{1 / 2}}^{\beta} J_{1}(x, q) d q\right| & =\left|\int_{\tau_{0}}^{\tau_{1}} Q^{\prime}(\tau)\left(Q^{2}(\tau)+x\right)^{-1 / 2} \sin \left(\left(x^{1 / 2} / \varepsilon\right) \tau^{-1}\right) d \tau\right| \\
\leq & \left|\int_{\tau_{0}}^{\tau_{1}}\left(1 / d_{1}\right)\left(\left(\tau / d_{1}\right)^{2}+x\right)^{-1 / 2} \sin \left(\left(x^{1 / 2} / \varepsilon\right) \tau^{-1}\right) d \tau\right| \\
& +\left|\int_{\tau_{0}}^{\tau_{1}} f_{1}(\tau)\left(\left(\tau / d_{1}\right)^{2}+x\right)^{-1 / 2} \sin \left(\left(x^{1 / 2} / \varepsilon\right) \tau^{-1}\right) d \tau\right| \\
= & I_{1}+I I_{1},
\end{aligned}
$$

where $f_{1}(\tau) \sim O(\tau)$ as $\tau \rightarrow 0$ and is bounded. But

$$
I_{1}=\left|\int_{x^{1 / 2} \tau_{0}^{-1}}^{x^{1 / 2} \tau_{1}^{-1}}\left(1 / d_{1}\right)\left(\left(1 / d_{1}\right)^{2}+y^{2}\right)^{-1 / 2} y^{-1} \sin (y / \varepsilon) d y\right| \leq K
$$

and

$$
I I_{1} \leq K_{1}\left|\tau_{1}-\tau_{0}\right| \leq K
$$

where $K$ and $K_{1}$ are fixed constants. Thus

$$
\left|\int_{\alpha_{1} x^{1 / 2}}^{\beta} J_{1}(x, q) d q\right| \leq K \text { for small } x .
$$

Then

$$
\left|\int_{0}^{\alpha x^{1 / 2}} J_{1}(x, q) d q\right| \leq \int_{0}^{\alpha x^{1 / 2}}\left(q^{2}+x\right)^{-1 / 2} d q \leq K
$$

and

$$
\left|\int_{\beta}^{d_{3}^{1 / 2}} J_{1}(x, q) d q\right| \leq(1 / \beta) d_{3}^{1 / 2} \leq K .
$$

Therefore, $|J| \leq K$ for $x<x_{0}$ with sufficiently small $x_{0}$ and $K$ is a fixed number. Also if $x \geq x_{0}>0$, then by the definition of $J$ and the stationary phase method, it is straightforward to show

$$
|J| \leq K\left(x_{0}\right) \varepsilon^{1 / 2}
$$


where $K\left(x_{0}\right)$ depends on $x_{0}$ only. Thus,

$$
\begin{gathered}
\left|\int_{-R}^{R} D(x, p) d p\right| \leq K \text { for all } x, \\
\left|\int_{-R}^{R} D(x, p) d p\right| \leq K\left(x_{0}\right) \varepsilon^{1 / 2} \text { for } x \geq x_{0} .
\end{gathered}
$$

From Eq. (28), we see that if $x \geq x_{0}$, then $|I(x)| \leq K\left(x_{0}\right) \varepsilon^{5 / 2}$ and $|I(x)| \leq K \varepsilon^{2}$ for all $x$. Hence $\phi_{1}(x, z)$ defined in Eq. (27) is an approximate solution of Eqs. (4)-(6) up to order $O\left(\varepsilon^{2}\right)$ for all $x$ and up to order $O\left(\varepsilon^{5 / 2}\right)$ for $x \geq x_{0}>0$.

Now we are in a position to show that $\lambda$ defined by Eq. (26) is also an approximate eigenvalue. Let

$$
\Omega=\{(x, z) \mid-\infty<x<+\infty, 0 \leq z \leq-h(x)\},
$$

and define an inner product and $L^{2}$-norm by

$$
(f, g)=\int_{\Omega} f g d x d z, \quad\|f\|^{2}=\int_{\Omega}|f|^{2} d x d z,
$$

and a Hilbert space $H$ by

$$
H=\{f \mid\|f\|<+\infty\} .
$$

Then by using the ideas in [15], we define an operator $\mathscr{A}$ with

$$
D(\mathscr{A})=\left\{f \in H \mid f \in H^{2}(\Omega), f_{z}+\varepsilon^{2} h_{x} f_{x}=0 \text { at } z=-h(x)\right\}
$$

where $H^{2}(\Omega)$ is a usual Sobolev space in $\Omega$ and

$$
\mathscr{A} f=-f_{z z}-\varepsilon^{2} f_{x x}+f .
$$

Define the operators $T$ and $\Gamma$ in $D(\mathscr{A})$ such that

$$
T f=\left.f_{z}(x, z)\right|_{z=0} \text { and } \Gamma f=\left.f(x, z)\right|_{z=0} .
$$

Let the restriction of $\mathscr{A}$ to the set of all $f$ in $D(\mathscr{A})$ for which $f_{z}(x, 0)=0$ be the operator $\mathscr{A}_{0}$. By using the Lax-Milgram theorem, it is not difficult to show that $\mathscr{A}_{0}$ is selfadjoint and positive with bounded inverse in $H$. Therefore, $\mathscr{A}_{0}^{1 / 2}$ is well defined. By a straightforward argument, these operators satisfy all the conditions stated in [15] except compactness of the operators. Therefore, if we define

$$
\mathscr{R}=\mathscr{A}_{0}^{1 / 2} T^{-1} \Gamma \mathscr{A}_{0}^{-1 / 2} \text { in } H,
$$

then $\mathscr{R}$ is selfadjoint and nonnegative. Equations (4)-(6) can be transformed into an operator equation

$$
\mathscr{R} \psi(x, z)=\lambda \psi(x, z)
$$

where $\psi \in H$, and if $\phi(x, z)$ is the generalized solution of Eqs. (4)-(6), then $\psi(x, z)=\mathscr{A}_{0}^{1 / 2} \phi(x, z)$. Thus by definition of $\phi_{1}(x, z)$, we know that $\phi_{1}(x, z)$ 
defined in Eq. (27) is an approximate solution of Eqs. (4)-(6) up to order $\varepsilon^{2}$ in the sup-norm in $\Omega$. From Eqs. (9) and (28), at $z=-h(x)$

$$
\begin{aligned}
I(x) & =\phi_{1 z}+\varepsilon^{2} h_{x} \phi_{1 x} \\
& =-\varepsilon^{2} \int_{C} B(x, p)\left(p^{2}+1\right)^{-1 / 2} \exp \left(i\left(p x-\left(\int_{0}^{p} P(p) d p+S_{0}\right)\right) / \varepsilon\right) d p .
\end{aligned}
$$

The critical point $p_{c}$ of $p x-\left(\int_{0}^{p} P(p) d p+S_{0}\right)$ satisfies $x-P\left(p_{c}\right)=0$ which implies

$$
\lambda=\left(p_{c}^{2}+1\right)^{1 / 2} \tanh \left(\left(p_{c}^{2}+1\right)^{1 / 2} h(x)\right)
$$

by the definition of $P(p)$. But $\lambda<\tanh h(\infty)$. So for large $x$, say $x \geq x_{0}$, $x=P\left(p_{c}\right)$ will have no real solution $p_{c}$. Therefore, if $x \geq 2 x_{0},|x-P(p)| \neq 0$ or equivalently $|x-P(p)| \geq K_{0}|x|$ for $p \in C$. By using integration by parts once, for $x \geq 2 x_{0}$

$$
\begin{aligned}
|I(x)|= & \left|\varepsilon^{2} \int_{C} B(x, p)\left(p^{2}+1\right)^{-1 / 2} \exp \left(i\left(p x-\left(\int_{0}^{p} P(p) d p+S_{0}\right)\right) / \varepsilon\right) d p\right| \\
= & \mid \varepsilon^{3} \int_{C} \frac{d}{d p}\left(B(x, p)\left(p^{2}+1\right)^{-1 / 2}(x-P(p))^{-1}\right) \\
& \times \exp \left(i\left(p x-\left(\int_{0}^{p} P(p) d p+S_{0}\right)\right) / \varepsilon\right) d p \mid \\
\leq & K_{2}|x|^{-1} \varepsilon^{3}
\end{aligned}
$$

since the function inside the integral over $C$ is of the order $\exp (-x|p| / 2)$ as $p$ goes to $+i \infty$, where $K_{2}$ is a constant independent of $\varepsilon$ and $x$. Finally using integration by parts $n$ times, we have that at $z=-h(x)$

$$
\left|\phi_{1 z}+\varepsilon^{2} h_{x} \phi_{1 x}\right| \leq K \varepsilon^{2}|x|^{-n} \text { for } x \geq 2 x_{0},
$$

where $n$ is any positive integer and $K$ is a constant independent of $\varepsilon$ and $x$. Therefore, if we can get an estimate in the sup-norm, the same estimate is true for the $L^{2}$-norm. Thus, by the definition of $\phi_{1}(x, z)$ in Eq. $(27)$, if $\psi_{1}(x, z)=$ $\mathscr{A}_{0}^{1 / 2} \phi_{1}(x, z)$, then

$$
\left|\left(\mathscr{R} \psi_{1}(x, z)-\lambda \psi_{1}(x, z), f(x, z)\right)\right| \leq K \varepsilon^{2}\|f\|
$$

for all $f \in H$, which implies

$$
\left\|\mathscr{R} \psi_{1}(x, z)-\lambda \psi_{1}(x, z)\right\| \leq K \varepsilon^{2} .
$$

Also by using the stationary phase method

$$
K \varepsilon^{1 / 2} \leq\left\|\phi_{1}(x, z)\right\| \leq\left\|\mathscr{A}_{0}^{1 / 2} \phi_{1}\right\|=\left\|\psi_{1}\right\| .
$$

Let us denote the spectrum of $\mathscr{R}$ by $\sigma(\mathscr{R})$, and let $d(\lambda, \sigma((\mathscr{R}))$ be the distance between $\lambda$ and $\sigma(\mathscr{R})$. Then by Lemma 13.1 in Maslov and Fedoriuk [14],

$$
d(\lambda, \sigma(\mathscr{R})) \leq K \varepsilon^{3 / 2},
$$

where $\lambda$ is defined by Eq. (26). Therefore, for small $\varepsilon$ we have that there is a $\lambda_{0}$ in the spectrum of Eqs. (4)-(6) so that $\lambda_{0}=\lambda+O\left(\varepsilon^{3 / 2}\right)$. Thus, $\lambda$ defined by Eq. (26) is an approximation of the spectrum of Eqs. (4)-(6) up to order $\varepsilon^{3 / 2}$. The approximate eigenfunction is $\phi_{1}(x, z)$ defined by Eq. (27). Finally, we summarize the results as 
THEOREM 1. The function defined by Eq. (27) is an approximate solution of Eqs. (4)(6) up to the order $\varepsilon^{2}$ for all $x>0$ and up to the order $\varepsilon^{5 / 2}$ for $x \geq x_{0}>0$, and the distance between $\lambda$ defined by Eq. (26) and the spectrum of Eqs. (4)-(6) is of the order $\varepsilon^{3 / 2}$.

Note that in this theorem we have to assume that the depth profile $H(x)$ depends on $x$ only, which means that the bottom of the beach is uniform in the $y$-direction.

5. Discussion. First let us show that the formulas used by Shen, Meyer, and Keller in [7] can be derived from Eqs. (26) and (27). From Eq. (27), if $x \geq x_{0}$, by using the stationary phase method,

$$
\begin{aligned}
\phi_{1}(x, z) \sim & \sum_{j=1}^{2} \exp \left(i\left(x p-\left(\int_{0}^{p} P(p) d p+S_{0}\right)\right) / \varepsilon\right) a_{0}\left(p^{2}+1\right)^{1 / 2} A(p) \\
& \times\left.\left(\cosh (\kappa z)+\frac{\sinh }{(\kappa h(x))} \cosh (\kappa h(x)) \sinh (\kappa x)\right)\right|_{p=g_{j}(x)},
\end{aligned}
$$

where $g_{j}(x), j=1,2$, are two roots of $P(p)=x$ for $p$. Thus,

$$
\begin{aligned}
\phi_{1}(x, z) & \sim \sum_{j=1}^{2}\left(\exp \left(i\left(x g_{j}(x)-\left(\int_{0}^{g_{j}} P(p) d p+S_{0}\right)\right) / \varepsilon\right) a_{0}\left(g_{j}^{2}+1\right)^{-1 / 2} A\left(g_{j}\right)\right. \\
& \times \cosh ^{-1}\left(\left(g_{j}^{2}+1\right)^{1 / 2} h(x)\right) \cosh \left(\left(g_{j}^{2}+1\right)^{1 / 2}(z+h(x))\right) .
\end{aligned}
$$

But if $k(x)=\left(g_{j}^{2}+1\right)^{1 / 2}$ and $T(x)=x g_{j}(x)-\left(\int_{0}^{g_{j}} P(p) d p+S_{0}\right)$, then $T_{x}=g_{j}(x)$, $T_{x}^{2}+1=k^{2}(x)$, and by (12)

$$
k(x) \tanh (k(x) h(x))=\lambda .
$$

These expressions for $T_{x}$ and $k(x)$ correspond to Eqs. (22)-(24) in [7]. Also by Eq. (26), $P(p)=x$, and letting $a$ be $P(0)$,

$$
\begin{aligned}
(2 n+1) \pi & =\int_{-\infty}^{+\infty} P(p) d p / \varepsilon=2 \int_{0}^{+\infty} P(p) d p / \varepsilon \\
& =(2 / \varepsilon)\left(\left.p P(p)\right|_{0} ^{+\infty}-\int_{0}^{+\infty} p P^{\prime}(p) d p\right) \\
& =(2 / \varepsilon)\left(-\int_{a}^{0} g_{1}(x) d x\right)=(2 / \varepsilon) \int_{0}^{1}\left(k^{2}(x)-1\right)^{1 / 2} d x,
\end{aligned}
$$

which is the formula (31) in [7].

Next we show that the uniform expansion of $\phi(x, z)$ in [11] can be derived from Eq. (27). From Eq. (27) we have

$$
\begin{aligned}
\phi_{1}(x, z)= & \int_{C_{R}} \exp \left(i\left(p x-\left(\int_{0}^{p} P(p) d p+S_{0}\right)\right) / \varepsilon\right) a_{0}\left(p^{2}+1\right)^{-1 / 2} A(p) \\
& \times\left(\cosh (\kappa z)+\lambda \kappa^{-1} \sinh (\kappa z)\right) d p
\end{aligned}
$$

with $x R^{2}=d_{3}$. Since $\kappa=\left(p^{2}+1\right)^{1 / 2}$, we let

$$
\begin{gathered}
\cosh \left(\left(p^{2}+1\right)^{1 / 2} z\right)+\lambda\left(p^{2}+1\right)^{-1 / 2} \sinh \left(\left(p^{2}+1\right)^{1 / 2} z\right) \\
=A_{1}(x, z)+(x-P(p)) A_{2}(p, z, x),
\end{gathered}
$$


where $A_{2}(p, z, x)$ is bounded on $C_{R}$,

$$
\begin{aligned}
A_{1}(x, z)= & \cosh \left(\left(p^{2}+1\right)^{1 / 2} z\right)+\left.\lambda\left(p^{2}+1\right)^{-1 / 2} \sinh \left(\left(p^{2}+1\right)^{1 / 2} z\right)\right|_{p=p^{+}} \\
& +\cosh \left(\left(p^{2}+1\right)^{1 / 2} z\right)+\left.\lambda\left(p^{2}+1\right)^{-1 / 2} \sinh \left(\left(p^{2}+1\right)^{1 / 2} z\right)\right|_{p=p^{-}},
\end{aligned}
$$

and $p^{+}, p^{-}$are the roots of $x=P(p)$. By integration by parts and letting $R \rightarrow+\infty$ for the first term, Eq. (31) becomes

$$
\begin{aligned}
& \phi_{1}(x, z) \\
& =A_{1}(x, z) \int_{C_{R}} \exp \left(i\left(p x-\left(\int_{0}^{p} P(p) d p+S_{0}\right)\right) / \varepsilon\right) a_{0}\left(p^{2}+1\right)^{-1 / 2} A(p) d p+O(\varepsilon) \\
& =A_{1}(x, z) \int_{-\infty}^{+\infty} \exp \left(i\left(p x-\left(\int_{0}^{p} P(p) d p+S_{0}\right)\right) / \varepsilon\right) a_{0}\left(p^{2}+1\right)^{-1 / 2} A(p) d p+O(\varepsilon) \\
& =2 A_{1}(x, z) e^{i S_{0} / \varepsilon} \int_{0}^{+\infty} \cos \left(\left(p x-\int_{0}^{p} P(p) d p\right) / \varepsilon\right) a_{0}\left(p^{2}+1\right)^{-1 / 2} A(p) d p+O(\varepsilon) .
\end{aligned}
$$

We use the following change of variable:

$$
p x+\int_{p}^{+\infty} P(p) d p=Q^{-2}(x) q+(1 / q)
$$

where $Q(x)$ is the positive root of $P(p)=x$; then $d p / d q \neq 0$, and choose $q$ so that $d p / d q>0$. Let $p=v(q)$ and $q=V(p)$. Note that $v(q)$ and $V(p)$ may depend on $x$. Then

$$
\begin{aligned}
\phi_{1}(x, z)= & 2 A_{1}(x, z) \exp \left(i S_{0} / \varepsilon\right) \\
& \times \int_{V(0)}^{+\infty} \cos \left(\left(Q^{-2}(x) q+(1 / q)-\int_{V(0)}^{+\infty} P(p) d p\right) / \varepsilon\right) \\
& \times a_{0}\left(v^{2}(q)+1\right)^{-1 / 2} B(q) d q,
\end{aligned}
$$

where $V(0) \geq$ const $>0$ for small $x$ and $B(q)=A(v(q))$ is bounded. Let

$$
\left(v^{2}(q)+1\right)^{-1 / 2} B(q)=q^{-1} B_{1}(q, x),
$$

and $B_{1}(q, x)$ is bounded since $q \sim x Q^{2}(x) p$ for $p$ large. Write $B_{1}(q, x)$ as

$$
B_{1}(q, x)=C_{0}(x)+(1 / q) C_{1}(x)+\left(Q^{-2}(x)-\left(1 / q^{2}\right)\right) C_{2}(x, q),
$$

where the derivative of $C_{2}(x, q)$ in terms of $q$ has order $\left(1 / q^{2}\right)$. Hence by using integration by parts,

$$
\begin{aligned}
\phi_{1}(x, z)= & A_{1}(x, z) \exp \left(i S_{0} / \varepsilon\right) \\
\times & \left(\int_{V(0)}^{+\infty}\left(C_{0}(x)+(1 / q) C_{1}(x)\right)\right. \\
& \left.\quad \times \cos \left(\left(Q^{-2}(x) q+(1 / q)\right) / \varepsilon-n \pi-(\pi / 2)\right) q^{-1} d q\right)+O(\varepsilon) .
\end{aligned}
$$


But

$$
\begin{aligned}
\int_{V(0)}^{+\infty} & \cos \left(\left(Q^{-2}(x) q+(1 / q)\right) / \varepsilon-n \pi-(\pi / 2)\right) q^{-1} d q \\
& =\int_{V(0) \varepsilon}^{+\infty} \sin \left((Q(x) \varepsilon)^{-2} q+(1 / q)\right)(-1)^{n} q^{-1} d q \\
& =\int_{0}^{+\infty}(-1)^{n} q^{-1} \sin \left(\left(Q^{2}(x) \varepsilon^{2}\right)^{-1} q+(1 / q)\right) d q+O(\varepsilon) \\
& =(-1)^{n} \pi J_{0}\left(2(Q(x) \varepsilon)^{-1}\right)+O(\varepsilon),
\end{aligned}
$$

where $J_{0}(z)$ is the zeroth-order Bessel function of the first kind. Moreover,

$$
\begin{aligned}
\int_{V(0)}^{+\infty} & \cos \left(\left(Q^{-2}(x) q+(1 / q)\right) / \varepsilon-n \pi-(\pi / 2)\right) q^{-2} d q \\
= & \varepsilon \int_{V(0) \varepsilon}^{+\infty}(-1)^{n} \sin \left(\left(Q^{2}(x) \varepsilon^{2}\right)^{-1} q+(1 / q)\right) q^{-2} d q \\
= & \varepsilon \int_{V(0) \varepsilon}^{1}(-1)^{n} \sin \left(\left(Q^{2}(x) \varepsilon^{2}\right)^{-1} q+(1 / q)\right) q^{-2} d q+O(\varepsilon) \\
= & \varepsilon \int_{V(0) \varepsilon}^{1}(-1)^{n+1}\left(\sin \left(\left(Q^{2}(x) \varepsilon^{2}\right)^{-1} q\right) \cos (1 / q)\right. \\
& \left.+\cos \left(\left(Q^{2}(x) \varepsilon\right)^{-1} q\right) \sin (1 / q)\right) d(1 / q)+O(\varepsilon) \\
= & Q^{-2}(x)(-1)^{n} \int_{V(0) \varepsilon}^{1} \sin \left(\left(Q^{2}(x) \varepsilon^{2}\right)^{-1} q+(1 / q)\right) d q+O(\varepsilon)
\end{aligned}
$$

Finally, by Eq. (32)

$$
\begin{aligned}
\phi_{1}(x, z)= & A_{1}(x, z) \exp \left(i S_{0} / \varepsilon\right)(-1)^{n}\left(\pi J_{0}\left(2(Q(x) \varepsilon)^{-1}\right) C_{0}(x)\right. \\
& \left.+C_{1}(x) Q^{-2}(x) \int_{0}^{1} \sin \left((Q(x) \varepsilon)^{-2} q+(1 / q)\right) d q\right)+O(\varepsilon),
\end{aligned}
$$

where $O(\varepsilon)$ is uniformly small with order $\varepsilon$ for $x$ near zero. By noting that $Q^{-2}(x) \sim\left(x / d_{1}\right)$ for small $x$, we have that if $Q^{2}(x) \varepsilon^{2} \sim O(1)$ or $Q^{2}(x) \varepsilon^{2} \gg O(1)$, which means that $x$ is comparable or smaller than $\varepsilon^{2}$, then $Q^{-2}(x) \leq O\left(\varepsilon^{2}\right)$ and

$$
\left.Q^{-2}(x) \int_{0}^{1} \sin \left(\left(Q^{2}(x) \varepsilon\right)^{-1} q+1 / q\right)\right) d q \sim O\left(\varepsilon^{2}\right) .
$$

So the first term in Eq. (33) is the dominant term. If $Q^{2}(x) \varepsilon^{2} \ll O(1)$, which means that $x$ is larger than $\varepsilon^{2}$, then by using the stationary phase method,

$$
\begin{gathered}
\pi J_{0}\left(2(Q(x) \varepsilon)^{-1}\right) C_{0}(x)+C_{1}(x) Q^{-2}(x) \int_{0}^{1} \sin \left((Q(x) \varepsilon)^{-2} q+(1 / q)\right) d q \\
\sim C_{0}^{*}(x) J_{0}\left(2(Q(x) \varepsilon)^{-1}\right)+C_{1}^{*}(x) J_{1}\left(2(Q(x) \varepsilon)^{-1}\right)+O(\varepsilon),
\end{gathered}
$$

where $C_{0}^{*}(x)$ and $C_{1}^{*}(x)$ are two new functions only dependent on $x$. Since Eq. (33) is valid uniformly for all $x<K$ with $K$ as a small constant independent of $\varepsilon$, by 
the above discussion we may assume $\phi_{1}(x, z)$ in Eq. (33) has the following form uniformly in $x$ :

$$
\begin{aligned}
\phi_{1}(x, z)=\exp \left(i S_{0}(x) / \varepsilon\right) & \left(D_{1}(x, z) J_{0}\left(2(Q(x) \varepsilon)^{-1}\right)\right. \\
& \left.+D_{2}(x, z) J_{1}\left(2(Q(x) \varepsilon)^{-1}\right)\right)+O(\varepsilon) .
\end{aligned}
$$

$D_{2}(x, z)$ could be zero when $x$ is zero and

$$
\left.D_{2}(x, z) J_{1}(Q(x) \varepsilon)^{-1}\right) \sim O\left(\varepsilon^{2}\right)
$$

if $Q(x) \varepsilon \sim O(1)$ or $Q(x) \varepsilon \gg O(1)$. Here $D_{1}(x, z)$ and $D_{2}(x, z)$ are two unknown functions. Thus, to obtain a uniform expansion of the solution of Eqs. (4)-(6), Eq. (34) is an appropriate form of the solution at least up to the first order. Equation (34) is the form used by Shen and Keller in [11] to derive the solution for more general situations.

Finally let us discuss the validity of the eigenvalue equation (26). Note that when we write Eqs. (12) and (13), we already use the fact that $\lambda \sim O(1)$ implicitly since we retain the terms $\lambda \cosh (\kappa h(x))$ and $\lambda \kappa^{-1} \sinh (\kappa h(x))$ in Eqs. (12) and (13) respectively. If we want to have $\lambda \sim O(\varepsilon)$, then Eqs. (12) and (13) are incorrect and the justification fails. Therefore, from the derivation, the assumption $\lambda \sim O(1)$ is necessary and by Eq. (26), $(2 n \pi+\pi / 2) \varepsilon \sim O(1)$ which implies that $n \sim O(1 / \varepsilon)$. But surprisingly enough, the formula works very well for $\lambda \sim O(\varepsilon)$ as well. Zhevandrov [12] uses the ray method to obtain higher-order terms of $\lambda$ and finds that for small $n$ the eigenvalue expansion is still correct by comparing his results with Miles's results [13] which are valid for $\lambda \sim O(\varepsilon)$. The extended range of the applicability of the eigenvalue formula has been recently justified by Zhevandrov [16].

Acknowledgment. We would like to thank Dr. Zhevandrov for his valuable help during the completion of this paper. The research reported here was partly supported by the National Science Foundation under Grant No. CMS-8903083.

\section{REFERENCES}

[1] F. Ursell, Edge waves on a sloping beach, Proc. Roy. Soc. London Ser. A 214, 79-97 (1952)

[2] A. S. Peters, Water waves over sloping beaches and the solution of a mixed boundary value problem for $\Delta \varphi-k^{2} \varphi=0$ in a sector, Comm. Pure Appl. Math. 5, 81-108 (1952)

[3] M. Roseau, Contribution à la théorie des ondes liquids de gravité en profondeur variable, Publications Scientifiques et Techniques du Ministère de l'Air, no. 275, Paris, 1952

[4] J. J. Stoker, Water Waves, Interscience, New York, 1957

[5] J. J. Stoker, The formation of breakers and bores, Comm. Pure Appl. Math. 1, 1-87 (1948)

[6] J. B. Keller, Surface waves on water of non-uniform depth, J. Fluid Mech. 4, 607-614 (1958)

[7] M. C. Shen, R. E. Meyer, and J. B. Keller, Spectra of water waves in channels and around islands, Phys. Fluids 11, 2289-2304 (1968)

[8] J. B. Keller and S. I. Rubinow, Asymptotic solution of eigenvalue problems, Ann. Phys. 9, 24-75 (1960)

[9] Yu. A. Kravtsov, A modification of the geometrical optics method, Radiofizika 7, 664-673 (1964)

[10] D. Ludwig, Uniform asymptotic expansions at a caustic, Comm. Pure. Appl. Math. 19, 215-250 (1966)

[11] M. C. Shen and J. B. Keller, Uniform ray theory of surface, internal and acoustic wave propagation in a rotating ocean or atmosphere, SIAM J. Appl. Math. 28, 857-875 (1975) 
[12] P. Zhevandrov, Edge waves on a gently sloping beach: Uniform asymptotics, J. Fluid Mech. 233, 483-493 (1991)

[13] J. W. Miles, Edge waves on a gently sloping beach, J. Fluid Mech. 199, 125-131 (1989)

[14] V. P. Maslov and M. V. Fedoriuk, Semi-classical approximation in quantum mechanics, D. Reidel, Boston, MA, 1981

[15] N. G. Askerov, S. G. Krein, and G. L. Laptev, A class of non-self-adjoint boundary value problems, Soviet Math. Dokl. 5, 424-427 (1964)

[16] P. Zhevandrov, Private communication, 1991 